\title{
EM-Based Receiver Design for Uplink MIMO-OFDMA Systems
}

\author{
Meng Wang, Graham C. Goodwin and Daniel E. Quevedo \\ School of Electrical Engineering \& Computer Science \\ The University of Newcastle, Callaghan, NSW 2308, Australia \\ Emails: MWang@studentmail.newcastle.edu.au,Graham.Goodwin@newcastle.edu.au,dquevedo@iee.org
}

\begin{abstract}
In this paper we propose an iterative receiver for uplink MIMO-OFDMA systems based on the expectation maximization (EM) algorithm. Iterating between the E-step and the M-step, the EM-based receiver updates the channel estimates and, refines data detection by increasing the likelihood function. Practical implementation issues are also considered: space-time block-coding (STBC) is incorporated to improve system performance against fading; a reduced-complexity algorithm is proposed, which simplifies the computation whilst not compromising performance.
\end{abstract}

\section{INTRODUCTION}

Orthogonal frequency division multiple access (OFDMA) has been proposed as a promising multiple access scheme for broadband wireless networks, e.g., IEEE 802.16 [1]. In OFDMA systems, the multiple access is realized by assigning mutually exclusive subsets of available subcarriers to different users for simultaneous data transmission. The orthogonality of subcarriers eliminates intra-cell interference and allows simple receiver implementation. Furthermore, OFDMA systems offer high flexibility in radio resource management according to the quality of service (QoS) demands of different users. By employing multiple antennas at both transmitter and receiver side, MIMO systems can increase channel capacity and mitigate the effect of multipath fading [2,3]. The combination of OFDMA and MIMO techniques, MIMO-OFDMA, has become a strong candidate for next-generation wireless networks.

In uplink MIMO-OFDMA systems, coherent signal detection at the base station (BS) requires the channel state information (CSI) of all uplink channels between the BS and mobile users. To obtain the CSI, channel estimators are designed at the BS receiver using pilot symbols. Due to the time-varying nature of multipath fading channels, the CSI has to be updated continuously and promptly, e.g., [4]. Notwithstanding, pilotbased receivers have the following shortcomings:

1) Considerable data rate is potentially sacrificed for channel estimation in multipath fading environments.

2) Error floors may be introduced by CSI estimate errors, especially for fast fading channels.

To deal with the above shortcomings, non pilot-aided, blind receiver designs have been reported in the literature (see [5] and references therein). However, blind receiver design suffers from several disadvantages including long data records and high complexity. Thus, there has been increasing interest in iterative receiver design, where joint channel estimation and data detection are performed iteratively. The expectation maximization (EM) algorithm [6, 7] has been employed in iterative receivers to perform maximum likelihood (ML) detection asymptotically with practical complexity. In the literature, EMbased receiver has been considered for single-user MIMO [8] and MIMO-OFDM systems $[9,10]$.

In this paper, we consider the design of an EM-based iterative receiver for uplink MIMO-OFDMA systems. The proposed receiver design can deal with flexible subcarrier allocation schemes [11] and approaches the ML receiver performance with limited complexity. For each user with allocated subcarriers, the proposed algorithm consists of two steps: E-step and M-step. The E-step performs channel estimation based upon hard decisions, assuming that the estimated data are known and correct. In the M-step, data detection is performed. The task can be split into several subproblems on a per-tone basis due to the orthogonality between subcarriers. To reduce the receiver complexity for implementation, we propose an inner-loop EM algorithm to avoid frequent inversion and multiplication of large matrices.

\section{SYSTEM MODEL}

We consider an uplink OFDMA system with one BS and $U$ mobile users. The BS and each user are equipped with $N_{R}$ and $N_{T}$ antennas, respectively. We assume that $N$ subcarriers are shared by all users and $N_{u}$ subcarriers are assigned exclusively to the $u$ th user. The subcarriers are allocated dynamically through an allocation algorithm [11]. The index set of the subcarriers allocated to the $u$ th user is given by

$$
I_{u}=\left\{i_{1}, i_{2}, \cdots, i_{N_{u}}\right\}, \quad u=1, \cdots, U .
$$

At the $u$ th user's transmitter, the $N_{u}$-length data symbol transmitted on the $t$ th antenna $S^{t, u}=\left\{S_{k}^{t, u}\right\}$ is mapped into an $N$-length data symbol $X^{t, u}=\left\{X_{k}^{t, u}\right\}$ by

$$
X_{k}^{t, u}=\left\{\begin{array}{cc}
S_{k}^{t, u}, & k \in I_{u} \\
0, & k \notin I_{u}
\end{array} \quad k=1, \cdots, N .\right.
$$

Then $X_{u}^{t}$ is processed by an inverse fast Fourier transform (IFFT) unit. Before transmission, an $N_{G}$-length cyclic prefix (CP) is appended to the IFFT output. The time-domain signal transmitted on the $t$ th antenna is given by

$$
x_{n}^{t, u}=\frac{1}{\sqrt{N}} \sum_{k \in I_{u}} X_{k}^{t, u} e^{\frac{j 2 \pi k n}{N}}, \quad-N_{G} \leq n \leq N-1
$$


We assume that the uplink channels between any pair of the user's transmit antenna and the BS receive antenna are independent and subject to uncorrelated multipath fading.

At the receiver side, if we assume perfect timing and frequency synchronization, the sampled signal received at the $r$ th antenna is given by

$$
y_{n}^{r}=\sum_{u=1}^{U} \sum_{t=1}^{N_{T}} \sum_{l=0}^{L} h_{l}^{r, t, u} x_{n-l}^{t, u}+w_{n}^{r}
$$

where $h_{l}^{r, t, u}$ is the $l$ th channel tap between the $r$ th BS receive antenna and the $u$ th user's $t$ th transmit antenna. $L$ denotes the maximum channel length. $w_{n}^{r}$ is additive white Gaussian noise (AWGN) with variance $\sigma_{w}^{2}$. After CP removal, the remaining samples are processed by a fast Fourier transform (FFT) unit. The FFT output at the $r$ th antenna is given by

$$
Y_{k}^{r}=\sum_{u=1}^{U} \sum_{t=1}^{N_{T}} H_{k}^{r, t, u} X_{k}^{t, u}+W_{k}^{r}, \quad k=1, \cdots, N .
$$

where $W_{k}^{r}$ is the FFT transform of $w_{n}^{r} . H_{k}^{r, t, u}$ denotes the channel frequency response on the $k$ th subcarrier, i.e.,

$$
H_{k}^{r, t, u}=\sum_{l=0}^{L-1} h_{l}^{r, t, u} \exp \left(\frac{-j 2 \pi k l}{N}\right)
$$

Considering that each subcarrier is assigned exclusively to one user, the FFT output for the $u$ th user can be written as

$$
Y_{k}^{r, u}=\sum_{t=1}^{N_{T}} H_{k}^{r, t, u} X_{k}^{t, u}+W_{k}^{r, u}, \quad k \in I_{u}
$$

Collecting the received samples on the $N_{u}$ subcarriers allocated to the $u$ th user, we have

$$
Y^{r, u}=\bar{X}^{u} H^{r, u}+W^{r, u}=\bar{X}^{u} \bar{F}^{u} h^{r, u}+W^{r, u}
$$

where

$$
\begin{aligned}
Y^{r, u} & =\left[Y_{i_{1}}^{r, u}, \cdots, Y_{i_{N_{u}}}^{r, u}\right]^{T} \in \mathbb{C}^{N_{u} \times 1} \\
\bar{X}^{u} & =\left[X^{1, u}, \cdots, X^{N_{T}, u}\right] \in \mathbb{C}^{N_{u} \times\left(N_{T} N_{u}\right)} \\
X^{t, u} & =\operatorname{diag}\left\{X_{i_{1}}^{t, u}, \cdots, X_{i_{N_{u}}}^{t, u}\right\} \in \mathbb{C}^{N_{u} \times N_{u}} \\
H^{r, u} & =\left[\left(H^{r, 1, u}\right)^{T}, \cdots,\left(H^{r, N_{T}, u}\right)^{T}\right]^{T} \in \mathbb{C}^{N_{T} N_{u} \times 1} \\
H^{r, t, u} & =\left[H_{i_{1}}^{r, t, u}, \cdots, H_{i_{N_{u}}}^{r, t, u}\right]^{T} \in \mathbb{C}^{N_{u} \times 1}
\end{aligned}
$$

In (8), $W^{r, u}$ is an $N_{u} \times 1$ circularly symmetric complex Gaussian noise vector and $\bar{F}^{u}$ is a block diagonal matrix with

$$
\begin{aligned}
\bar{F}^{u} & =\operatorname{diag}\left\{F^{u}, \cdots, F^{u}\right\} \in \mathbb{C}^{N_{T} N_{u} \times N_{T} L} \\
F^{u}(k, l) & =\exp \left(\frac{-j 2 \pi k l}{N}\right), \quad l=0, \cdots, L-1 .
\end{aligned}
$$

We note that $F^{u}$ is a submatrix of the FFT matrix, obtained by selecting $N_{u}$ rows according to $I_{u}$. $h^{r, u}$ contains all channel impulse responses between the $u$ th user and $r$ th BS antenna:

$$
\begin{aligned}
h^{r, u} & =\left[\left(h^{r, 1, u}\right)^{T}, \cdots,\left(h^{r, N_{T}, u}\right)^{T}\right]^{T} \in \mathbb{C}^{N_{T} L \times 1} \\
h^{r, t, u} & =\left[h_{0}^{r, t, u}, \cdots, h_{L-1}^{r, t, u}\right]^{T}, \quad t=1, \cdots, N_{T} .
\end{aligned}
$$

where $(\cdot)^{T}$ denotes transpose. Incorporating (8), we can write the received signals over all $N_{R}$ antennas as

$$
\bar{Y}^{u}=\bar{X}^{u} \bar{F}^{u} \bar{h}^{u}+\bar{W}^{u}
$$

where

$$
\begin{aligned}
\bar{Y}^{u} & =\left[Y^{1, u}, \cdots, Y^{N_{R}, u}\right] \in \mathbb{C}^{N_{u} \times N_{R}} \\
\bar{h}^{u} & =\left[h^{1, u}, \cdots, h^{N_{T}, u}\right] \in \mathbb{C}^{N_{T} L \times N_{R}} \\
\bar{W}^{u} & =\left[W^{1, u}, \cdots, W^{N_{R}, u}\right] \in \mathbb{C}^{N_{u} \times N_{R}}
\end{aligned}
$$

\section{EM-BASED RECEIVER DESIGN FOR UPLINK MIMO-OFDMA}

In this section, we will develop an EM-based receiver for uplink MIMO-OFDMA systems. We will show that it can track a time-varying channel and requires far less pilot symbols than conventional receivers.

\section{A. EM Algorithm}

Suppose that we wish to estimate a parameter $\theta$ from a set of observations $\mathcal{Y}$. Then, the ML estimate is given by

$$
\hat{\theta}=\arg \max _{\theta} f(\mathcal{Y} \mid \theta)
$$

In many practical situations, direct computation of (12) is of prohibitive complexity. To alleviate the computational burden, the EM algorithm [6,7] was introduced to find the ML solution iteratively. We denote the hidden variables by $\mathcal{X}$. Also, $\mathcal{Y}$ denotes the observed data (incomplete) and $\mathcal{Z}=(\mathcal{X}, \mathcal{Y})$ is the complete data. The EM algorithm consists of two step: E-step and M-step. Starting with an initial estimate $\theta^{(0)}$, the E-step calculates the expected value of the log-likelihood function with respect to the hidden variables conditioned on the observation and latest estimate:

$$
Q\left(\theta \mid \theta^{(j)}\right)=E\left\{\log f(\mathcal{Z} \mid \theta) \mid \mathcal{Y}, \theta^{(j)}\right\} .
$$

The M-step finds $\theta^{(j+1)}$ by maximizing (13) over $\theta$ :

$$
\theta^{(j+1)}=\arg \max _{\theta} Q\left(\theta \mid \theta^{(j)}\right)
$$

The E-step and M-step are computed alternatively. The EM algorithm is guaranteed to converge to the local maximum of the likelihood function. Thus, convergence to the global maximum depends on the initial estimate [7].

\section{B. Receiver Design Based on the EM Algorithm}

In an uplink MIMO-OFDMA system, we consider the channel impulse response $\bar{h}^{u}$ to be the hidden variables. Thus, we choose $\bar{Y}^{u}$ and $\left(\bar{Y}^{u}, \bar{h}^{u}\right)$ to be the incomplete data and complete data, respectively.

1) E-step: Given $\hat{X}^{u(j)}$, the E-step calculates the expectation of the log-likelihood function with respect to $\bar{h}^{u}$ conditioned on $\bar{Y}^{u}$ and $\hat{X}^{u(j)}$, that is,

$$
Q\left(\bar{X}^{u} \mid \hat{X}^{u(j)}\right)=E\left\{\log f\left(\bar{Y}^{u}, \bar{h}^{u} \mid \bar{X}^{u}\right) \mid \bar{Y}^{u}, \hat{X}^{u(j)}\right\}
$$

where $f\left(\bar{Y}^{u}, \bar{h}^{u} \mid \bar{X}^{u}\right)$ is the joint conditional probability density function for $\bar{Y}^{u}$ and $\bar{h}^{u}$ given $\bar{X}^{u}$. Assuming that the 
channel parameters $\bar{h}^{u}$ are independent of data symbols $\bar{X}^{u}$, it holds that

$$
\log f\left(\bar{Y}^{u}, \bar{h}^{u} \mid \bar{X}^{u}\right)=\log f\left(\bar{Y}^{u} \mid \bar{h}^{u}, \bar{X}^{u}\right)+\log f\left(\bar{h}^{u} \mid \bar{X}^{u}\right)
$$

where $f\left(\bar{h}^{u} \mid \bar{X}^{u}\right)=f\left(\bar{h}^{u}\right)$. Consequently, (15) is equivalent to finding

$$
Q^{\prime}\left(\bar{X}^{u} \mid \hat{X}^{u(j)}\right)=E\left\{\log f\left(\bar{Y}^{u} \mid \bar{h}^{u}, \bar{X}^{u}\right) \mid \bar{Y}^{u}, \hat{X}^{u(j)}\right\}
$$

Following the proof in [10], the expectation in (16) can be written in the form of

$$
\begin{aligned}
& Q^{\prime}\left(\bar{X}^{u} \mid \hat{X}^{u(j)}\right) \\
= & -\left\|\bar{Y}^{u}-\bar{X}^{u} \bar{F}^{u} \hat{h}^{u(j)}\right\|^{2}-\operatorname{Tr}\left\{\bar{X}^{u} \bar{F}^{u} \hat{\Sigma}^{u(j)} \bar{F}^{u^{H}} \bar{X}^{u^{H}}\right\}
\end{aligned}
$$

with

$$
\begin{gathered}
\hat{h}^{u(j)}=\frac{1}{\sigma_{w}^{2}} \hat{\Sigma}^{u(j)} \bar{F}^{u^{H}} \hat{X}^{u(j)^{H}} \bar{Y}^{u} \\
\hat{\Sigma}^{u(j)}=\left[\left(\Sigma^{u}\right)^{-1}+\frac{1}{\sigma_{w}^{2}} \bar{F}^{u^{H}} \hat{X}^{u(j)^{H}} \hat{X}^{u(j)} \bar{F}^{u}\right]^{-1}
\end{gathered}
$$

where $(\cdot)^{H}$ denotes conjugate transpose. Note that $\hat{h}^{u(j)}$ is essentially the minimum mean-square error (MMSE) estimate of $\bar{h}^{u}$ given $\bar{Y}^{u}$ and $\hat{X}^{u(j)}$. $\Sigma^{u}$ denotes the covariance matrix of $\bar{h}^{u}$. Assuming that the channels between different pairs of transmit and receive antennas are uncorrelated, $\Sigma^{u}$ is a diagonal matrix known at the receiver. Recalling the orthogonality between subcarriers, (17) can be rewritten as

$$
\begin{aligned}
& Q^{\prime}\left(\bar{X}^{u} \mid \hat{X}^{u(j)}\right) \\
= & -\sum_{k \in I_{u}}\left\{\left\|\bar{Y}_{k}^{u}-\hat{H}_{k}^{u(j)} \bar{X}_{k}^{u}\right\|^{2}+\bar{X}_{k}^{u^{H}} \hat{\Sigma}_{k}^{u(j)} \bar{X}_{k}^{u}\right\}(20)
\end{aligned}
$$

where $\hat{H}_{k}^{u(j)}$ denotes the estimated channel frequency response on the $k$ th subcarrier. $\hat{H}_{k}^{u(j)}$ can be calculated by Fourier transform of $\bar{h}^{u(j)}$, i.e., $\hat{H}^{u(j)}=\bar{F}^{u} \bar{h}^{u(j)} \cdot \hat{\Sigma}_{k}^{u(j)}$ is formed by selecting the corresponding entries from the matrix $\bar{F}^{u} \hat{\Sigma}^{u(j)} \bar{F}^{u^{H}}$ in (17).

2) M-step: The M-step consists in finding $\hat{X}^{u(j+1)}$ by

$$
\begin{aligned}
& \arg \max _{\bar{X}^{u}} Q^{\prime}\left(\bar{X}^{u} \mid \hat{X}^{u(j)}\right) \\
= & \arg \min _{\bar{X}^{u}} \sum_{k \in I_{u}}\left\{\left\|\bar{Y}_{k}^{u}-\hat{H}_{k}^{u(j)} \bar{X}_{k}^{u}\right\|^{2}+\bar{X}_{k}^{u^{H}} \hat{\Sigma}_{k}^{u(j)} \bar{X}_{k}^{u}\right\}
\end{aligned}
$$

The above equation indicates that the M-step can be performed on each subcarrier independently. On the $k$ th subcarrier,

$$
\hat{X}_{k}^{u(j+1)}=\arg \min _{\bar{X}_{k}^{u}}\left\|\bar{Y}_{k}^{u}-\hat{H}_{k}^{u(j)} \bar{X}_{k}^{u}\right\|^{2}+\bar{X}_{k}^{u^{H}} \hat{\Sigma}_{k}^{u(j)} \bar{X}_{k}^{u}
$$

We can rewrite (22) as

$$
\hat{X}_{k}^{u(j+1)}=\arg \min _{\bar{X}_{k}^{u}}\left\|\bar{Y}_{k}^{u^{\prime}}-A_{k} \bar{X}_{k}^{u}\right\|^{2}
$$

where $\bar{Y}_{k}^{u^{\prime}}=A_{k}^{-H} \hat{H}_{k}^{u(j)^{H}} \bar{Y}_{k}^{u}$ and

$$
A_{k}^{H} A_{k}=\hat{H}_{k}^{u(j)^{H}} \hat{H}_{k}^{u(j)}+\hat{\Sigma}_{k}^{u(j)}
$$

obtained by a Cholesky factorization. Note that the data detector proposed in (23) is an ML MIMO receiver. Clearly, the complexity of exhaustive search is prohibitive for large $N_{T}$ (antenna arrays) and/or higher order constellations. The development of sphere decoding techniques promises to reduce the decoding complexity significantly, see [12] for more details.

\section{Reduced-Complexity STBC RECEIVER DESIGN}

In this section, we consider practical issues when implementing the EM-based receiver. Space-time block-code (STBC) is employed to enhance the system performance by achieving maximum spatial diversity. A reduced-complexity STBC receiver is proposed for implementation.

\section{A. STBC applied to the EM receiver}

STBC [13] has been proposed for multiple-antenna systems to improve performance in a fading environment. Orthogonal design of STBCs can achieve the maximum diversity gain. We assume that each mobile user is equipped with $N_{T}=2$ transmit antennas and the Alamouti scheme [14] is applied. Given (9), the OFDM word at sample time $n$ is denoted by

$$
\bar{X}_{n}^{u}=\left[X_{n}^{1, u}, X_{n}^{2, u}\right]
$$

The Alamouti scheme gives the OFDM word by

$$
\bar{X}_{n+1}^{u}=\left[-X_{n}^{2, u^{H}}, X_{n}^{1, u^{H}}\right]
$$

Similar to (11), the system model for two consecutive words can be written as

$$
\overline{\mathbf{Y}}^{u}=\overline{\mathbf{X}}^{u} \bar{F}^{u} \bar{h}^{u}+\overline{\mathbf{W}}^{u}
$$

with $\overline{\mathbf{X}}^{u}=\left[\bar{X}_{n}^{u^{T}}, \bar{X}_{n+1}^{u^{T}}\right]^{T}, \overline{\mathbf{Y}}^{u}=\left[\bar{Y}_{n}^{u^{T}}, \bar{Y}_{n+1}^{u^{T}}\right]^{T}$ and $\overline{\mathbf{W}}^{u}=$ $\left[\bar{W}_{n}^{u^{T}}, \bar{W}_{n+1}^{u^{T}}\right]^{T}$. We assume that the uplink channels remain constant during one STBC code word. The EM receiver design proposed in Section III can be modified to the STBC scenario. The E-step can be expressed as

$$
\begin{aligned}
& Q^{\prime}\left(\overline{\mathbf{X}}^{u} \mid \hat{\mathbf{X}}^{u(j)}\right) \\
= & -\left\|\overline{\mathbf{Y}}^{u}-\overline{\mathbf{X}}^{u} \bar{F}^{u} \hat{h}^{u(j)}\right\|^{2}-\operatorname{Tr}\left\{\overline{\mathbf{X}}^{u} \bar{F}^{u} \hat{\Sigma}^{u(j)} \bar{F}^{u^{H}} \overline{\mathbf{X}}^{u^{H}}\right\}
\end{aligned}
$$

with

$$
\begin{gathered}
\hat{h}^{u(j)}=\frac{1}{\sigma_{w}^{2}} \hat{\Sigma}^{u(j)} \bar{F}^{u^{H}} \hat{\mathbf{X}}^{u(j)^{H}} \overline{\mathbf{Y}}^{u} \\
\hat{\Sigma}^{u(j)}=\left[\left(\Sigma^{u}\right)^{-1}+\frac{1}{\sigma_{w}^{2}} \bar{F}^{u^{H}} \hat{\mathbf{X}}^{u(j)^{H}} \hat{\mathbf{X}}^{u(j)} \bar{F}^{u}\right]^{-1}
\end{gathered}
$$

The M-step is as in (22).

\section{B. Reduced-complexity design with an EM-based inner loop}

In the E-step, see (30), frequent inversion and multiplication of large matrices are required. We next propose an alternative design that reduces complexity.

Given (29), if we neglect the small term $\sigma_{w}^{2}\left(\Sigma^{u}\right)^{-1}$, the MMSE channel estimate of $\hat{h}^{u}$ reduces to

$$
\hat{h}^{u(j)}=\left[\bar{F}^{u^{H}} \hat{\mathbf{X}}^{u(j)^{H}} \hat{\mathbf{X}}^{u(j)} \bar{F}^{u}\right]^{-1} \bar{F}^{u^{H}} \hat{\mathbf{X}}^{u(j)^{H}} \overline{\mathbf{Y}}^{u}
$$


which turns out to be a least square (LS) channel estimator. The corresponding optimization problem is given by

$$
\hat{h}^{u(j)}=\arg \min _{\bar{h}^{u}}\left\|\overline{\mathbf{Y}}^{u}-\overline{\mathbf{X}}^{u(j)} \bar{F}^{u} \bar{h}^{u}\right\|^{2}
$$

To avoid matrix inversion, we propose a secondary EM-based algorithm that is built in the E-step (28) as an inner loop to solve (32) iteratively. This algorithm can also be generalized to cases where $N_{T}>2$. Note that the channel estimation problem can be separated into $N_{R}$ independent multi-input single-output (MISO) optimization problems, i.e.,

$$
\hat{h}^{r, u(j)}=\arg \min _{h^{r, u}}\left\|\overline{\mathbf{Y}}^{r, u}-\overline{\mathbf{X}}^{u(j)} \bar{F}^{u} h^{r, u}\right\|^{2},
$$

because the uplink channels to the $r$ th BS antenna are independent of the ones to other antennas.

Note that the diversity gain obtained from STBC can also be used to enhance the performance of channel estimation. We reorganize $\overline{\mathbf{X}}^{u}$ in a block-diagonal form as

$$
\hat{\mathbf{X}}^{u}=\left[\operatorname{diag}\left(X_{n}^{1, u},-X_{n}^{2, u^{H}}\right), \operatorname{diag}\left(X_{n}^{2, u}, X_{n}^{1, u^{H}}\right)\right]
$$

Then (33) can be written as

$$
\hat{h}^{r, u(j)}=\arg \min _{h^{r, u}}\left\|\overline{\mathbf{Y}}^{r, u}-\hat{\mathbf{X}}^{u(j)} \hat{\mathbf{F}}^{u} h^{r, u}\right\|^{2},
$$

where

$$
\hat{\mathbf{F}}^{u}=\left[\begin{array}{cc}
\hat{F}^{u} & \mathbf{0} \\
\mathbf{0} & \hat{F}^{u}
\end{array}\right]
$$

with $\hat{F}^{u}=\left[F^{u}, F^{u}\right]^{T}$.

Applying the EM algorithm to solve (35), we regard $\overline{\mathbf{Y}}^{r, u}$ to be the incomplete data. Then we separate $\overline{\mathbf{Y}}^{r, u}$ into $N_{T}$ components and regard $Y^{t, r, u}$ as the contributions from the $t$ th transmit antenna on shaping $\overline{\mathbf{Y}}^{r, u}$ :

$$
\sum_{t=1}^{N_{T}} Y^{r, t, u}=\sum_{t=1}^{N_{T}} Z^{t, r, u}+\sum_{t=1}^{N_{T}} W^{r, t, u}
$$

where $Z^{t, r, u}=\hat{X}^{t, u} \hat{F}^{u} h^{r, t, u}$ denotes the signal component from the $t$ th transmit antenna. $W^{r, t, u}=\beta_{t} W^{r, u}$, denotes the noise distributed over all channels, satisfying $\sum_{t=1}^{N_{T}} \beta_{t}=1$.

We choose $\left\{Y^{1, r, u}, \cdots, Y^{N_{T}, r, u}\right\}$ to be the complete data. As a consequence, the inner-loop EM algorithm for solving (35) has the following two steps:

1) Inner-loop E-step: With the estimated $\hat{X}^{u(j)}$ obtained in (21), compute

$$
Z^{t, r, u\left(j^{\prime}+1\right)}=\hat{X}^{t, u(j)} \hat{F}^{u} h^{r, t, u\left(j^{\prime}\right)}
$$

where $j^{\prime}$ denotes the iteration number in the inner-loop. (38) updates the signal component from each transmit antenna. $Y^{t, r, u}$ is updated by

$$
Y^{t, r, u\left(j^{\prime}+1\right)}=Z^{t, r, u\left(j^{\prime}+1\right)}+\beta_{t}\left[\overline{\mathbf{Y}}^{r, u}-\sum_{t=1}^{N_{T}} Z^{t, r, u\left(j^{\prime}+1\right)}\right]
$$

2) Inner-loop M-step: Given $Y^{t, r, u\left(j^{\prime}+1\right)}$, (33) can be further simplified to a single-antenna estimation problem:

$$
\begin{aligned}
& \hat{h}^{t, r, u\left(j^{\prime}+1\right)} \\
= & \arg \min _{h^{t, r, u}}\left\|Y^{t, r, u\left(j^{\prime}+1\right)}-\hat{X}^{t, u(j)} \hat{F}^{u} h^{r, t, u}\right\|^{2} \\
= & {\left[\hat{F}^{u H} \hat{X}^{t, u(j)^{H}} \hat{X}^{t, u(j)} \hat{F}^{u}\right]^{-1} \hat{F}^{u H} \hat{X}^{t, u(j)^{H}} Y^{t, r, u\left(j^{\prime}+1\right)} } \\
= & {\left[\hat{F}^{u H} \hat{F}^{u}\right]^{-1} \hat{F}^{u H} \hat{X}^{t, u(j)^{H}} Y^{t, r, u\left(j^{\prime}+1\right)} }
\end{aligned}
$$

since $\hat{X}^{t, u(j)^{H}} \hat{X}^{t, u(j)}=\mathbf{I}_{N_{T} N_{d}}$. Note that $\left[\hat{F}^{u H} \hat{F}^{u}\right]^{-1}$ can be predetermined and thus, matrix inversion is avoided. The inner-loop E-step and M-step are repeated alternatively. The converged estimate is then used in the outer-loop iterations.

\section{Simulation Results}

We consider an uplink OFDMA system with $N=256$ subcarriers over a $20 \mathrm{MHz}$ bandwidth. The system can support $U=8$ users to communicate with the BS simultaneously. We focus on the \#3 user and $N_{d}=32$ subcarriers are allocated to this user. The user and the BS are equipped with $N_{T}=2$ transmit antennas and $N_{R}=2$ receive antennas, respectively. The Alamouti STBC is used and the modulator adopts a QPSK constellation. We consider three packet lengths: each data packet contains $P_{L}=5,8,10$ STBC words. We assume that the first word contains pilot symbols for initial channel estimation while other words in the packet contain only data. The packets are transmitted through the frequency-selective Rayleigh fading channels of order $L=3$ and with exponential power decay. Autoregressive (AR) stochastic model [15] is used for the simulation of the Rayleigh fading channels. The dynamics of the channel taps can be described as

$$
\bar{h}_{l}^{u}(t+1)=\beta_{l} \bar{h}_{l}^{u}(t)+\alpha_{l} \bar{g}_{l}, \quad l=0, \cdots, L-1
$$

where $\beta_{l}=J_{0}\left(2 \pi f_{D}(t)\right)$ and $\alpha_{l}=\sqrt{\left(1-\beta_{l}^{2}\right) e^{-l / 5}} . J_{0}(\cdot)$ and $f_{D}(t)$ denote the zero-order Bessel function of the first kind and the Doppler frequency respectively; $\bar{g}_{l}$ is a random matrix whose entries are complex Gaussian variable $N(0,1)$. The parameter $\alpha_{l}$ captures the time-varying nature of the channel. In our simulation, we choose $\alpha_{0}=0.199,0.312$. A rate $1 / 2$ four-state convolutional code is used.

We first assume that perfect CSI is available at the receiver side. The performance of the ML detector is used to compare with that of the proposed EM-based receiver. As shown in Fig.1, the STBC word error rate (WER) that the proposed receiver can achieve is very close to that of the optimal ML receiver. We also compare the performance of the proposed receiver with 1, 2 and 3 iterations in the EM algorithm, given different channel dynamics, i.e., $\alpha_{0}=0.199,0.312$. It can be observed that WER performance of the cases with 1 and 2 iterations approximates quickly to the 3 iterations case. We thus conclude that only a small number of iterations are required in the EM process to achieve near-ML performance. This suggests that the computational effort is not excessive. 


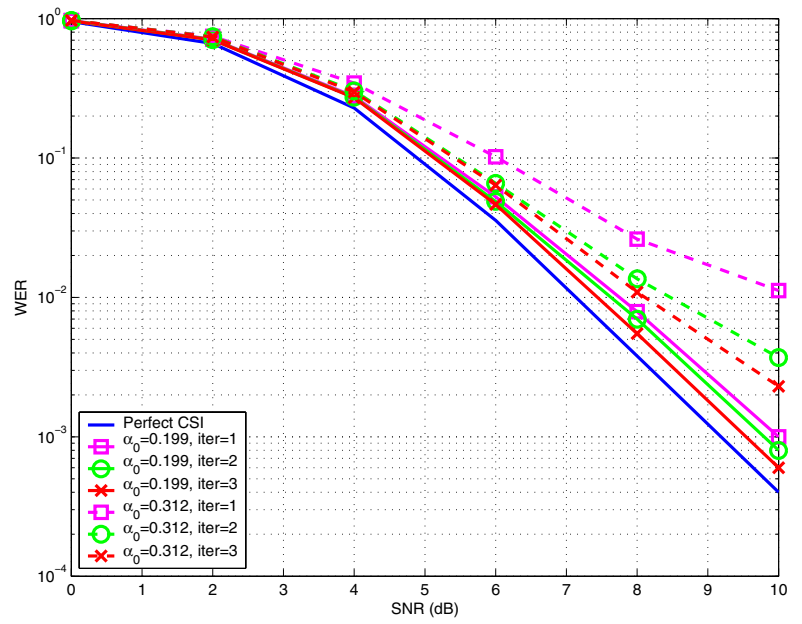

Fig. 1. WER performance of the proposed EM receiver with different number of iterations in the EM algorithm.

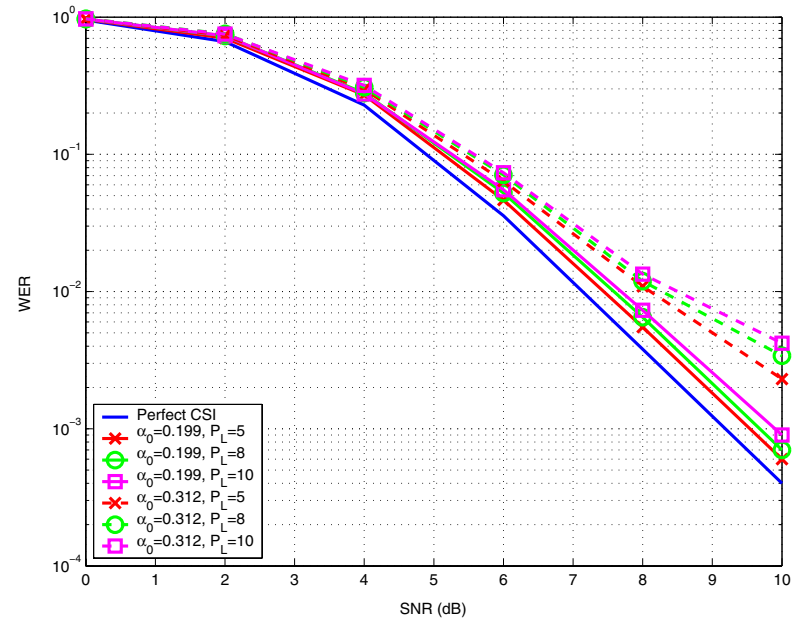

Fig. 2. WER performance of the proposed EM receiver with different number of STBC words contained in the packets.

We have assumed that only the first word in each data packet contains pilot symbols. To reduce the bandwidth loss, one needs to decrease the proportion of pilot symbols as much as possible. But longer data packets that contain STBC words may incur performance degradation due to the dynamic nature of the channels. To investigate this aspect, we simulate the OFDMA uplink transmission with one packet containing different number of STBC words. The results are illustrated in Fig.2, where we compare the WER performance with packets containing $P_{L}=5,8,10$ words. It can be seen that for Rayleigh fading channels $(\alpha=0.199,0.312)$, the WER performance does not degrade much when the packet length increases. Thus, the proposed receiver can capture the channel dynamics well with little spectral sacrifice.

In Fig.3, we simulate the performance of the reducedcomplexity receiver proposed in Section IV-B with only 2 iterations in the inner-loop EM. Compared with the original design, this reduced-complexity receiver can achieve close WER performance. Thus, the EM-based receiver can be implemented in practice with affordable computational cost.

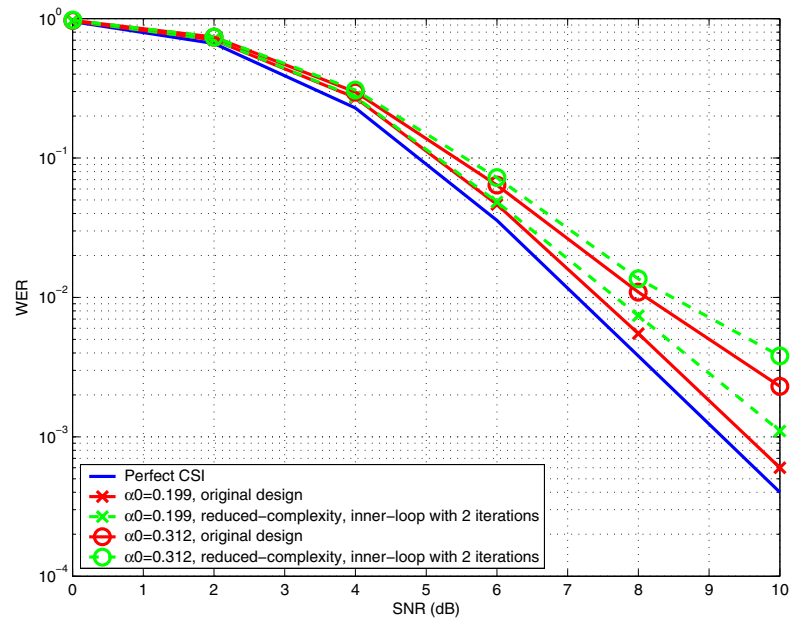

Fig. 3. WER performance of the reduced-complexity EM receiver with 2 iterations in the inner-loop EM process.

\section{CONClusions}

In this paper, we have presented an EM-based iterative receiver and its reduced-complexity version for uplink MIMOOFDMA systems. The proposed receiver performs joint data detection and channel estimation iteratively and can achieve comparable performance with that of a coherent detector with practical complexity.

\section{REFERENCES}

[1] IEEE Standard for Local and Metropolitan Area Networks Part 16: Air Interface for Fixed Broadband Wireless Access Systems. IEEE Standard 802.16, 2004.

[2] A. Paulraj, R. Nabar, and D. Gore. Introduction to Space-Time Wireless Communications. Cambridge University Press, UK, 2003.

[3] G. L. Stuber, J. R. Barry, S. W. McLaughlin, Ye Li, M. A. Ingram, and T. G. Pratt. Broadband MIMO-OFDM wireless communications. Proceedings of the IEEE, 92(2):271-294, February 2004.

[4] Yi Ma and R. Tafazolli. Channel estimation for OFDMA uplink: a hybrid of linear and BEM interpolation approach. IEEE Transactions on Signal Processing, 55(4):1568-1573, April 2007.

[5] Z. Ding and Y. Li. Blind Equalization and Identification. Marcel Dekker Inc., New York, 2000.

[6] A. P. Dempster, N. M. Laird, and D. B. Rubin. Maximum likelihood from incomplete data via the EM algorithm. J. Royal Stat. Soc., vol. 39, no. 1:1-38, Dec. 1977.

[7] G. J. McLachlan and T. Krishnan. The EM Algorithm and Extensions. John Wiley \& Sons, 1997.

[8] C. Cozzo and B. L. Hughes. Joint channel estimation and data detection in space-time communications. IEEE Transactions on Communications, Vol. 51:1266-1270, 2003.

[9] B. Lu, X. Wang, and Y. Li. Iterative receivers for space-time block-coded OFDM systems in dispersive fading channels. IEEE Trans. Wireless Commun., vol. 1, no. 2:213-225, Apr. 2002.

[10] M. Wang, D. E. Quevedo, and G. C. Goodwin. Joint data detection and channel estimation for MIMO-OFDM systems via EM algorithm and sphere decoding. In GLOBECOM '06, San Francisco, 2006.

[11] S. Pietrzyk. OFDMA for Broadband Wireless Access. Artech House Publishers, Norwood, MA, 2006.

[12] B. Hassibi and H. Vikalo. On the sphere-decoding algorithm I. Expected complexity. IEEE Trans. Signal Process., 53(8):2806-2818, Aug. 2005.

[13] V. Tarokh, H. Jafarkhani, and A. R. Calderbank. Space-time block codes from orthogonal designs. IEEE Transactions on Information Theory, vol. 45:1456-1467, 1999.

[14] S. M. Alamouti. A simple transmit diversity technique for wireless communications. IEEE Journal on Selected Areas in Communications, vol. 16:1451-1458, 1998.

[15] K. E. Baddour and N. C. Beaulieu. Autoregressive modeling for fading channel simulation. IEEE Transactions on Wireless Communications, 4(4):1650-1662, July 2005. 